\title{
Effects of a Dynamic Chair on Chair Seat Motion and Trunk Muscle Activity during Office Tasks and Task Transitions
}

\author{
Corina Nüesch ${ }^{1,2} \mathbb{D}$, Jan-Niklas Kreppke ${ }^{3}$, Annegret Mündermann ${ }^{1,2} \mathbb{D}$ and Lars Donath ${ }^{4, *}$ \\ 1 Department of Orthopaedics and Traumatology, University Hospital Basel, 4031 Basel, Switzerland; \\ corina.nueesch@usb.ch \\ 2 Department of Biomedical Engineering, University of Basel, 4123 Allschwil, Switzerland; \\ annegret.muendermann@unibas.ch \\ 3 Department of Sport, Exercise and Health, University of Basel, 4052 Basel, Switzerland; \\ jan-niklas.kreppke@stud.unibas.ch \\ 4 Department of Intervention Research in Exercise Training, German Sport University Cologne, \\ 50933 Köln, Germany \\ * Correspondence: 1.donath@dshs-koeln.de; Tel.: +49-2214-9827-700
}

Received: 5 November 2018; Accepted: 29 November 2018; Published: 3 December 2018

\begin{abstract}
Employing dynamic office chairs might increase the physical (micro-) activity during prolonged office sitting. We investigated whether a dynamic BioSwing ${ }^{\circledR}$ chair increases chair sway and alters trunk muscle activation. Twenty-six healthy young adults performed four office tasks (reading, calling, typing, hand writing) and transitions between these tasks while sitting on a dynamic and on a static office chair. For all task-transitions, chair sway was higher in the dynamic condition $(p<0.05)$. Muscle activation changes were small with lower mean activity of the left obliquus internus during hand writing $(p=0.07)$, lower mean activity of the right erector spinae during the task-transition calling to hand writing $(p=0.036)$, and higher mean activity of the left erector spinae during the task-transition reading to calling $(p=0.07)$ on the dynamic chair. These results indicate that an increased BioSwing ${ }^{\circledR}$ chair sway only selectively alters trunk muscle activation. Adjustments of chair properties (i.e., swinging elements, foot positioning) are recommended.
\end{abstract}

Keywords: dynamic sitting; office chair; muscle activation; inactivity; sedentarism

\section{Introduction}

Physical inactivity is considered an independent risk factor for many non-communicable diseases and has been entitled as biggest public health problem of the 21st century [1]. Projections have indicated that the metabolic equivalent (MET) of daily physical work will drop to 190 MET hours per week in 2030 because of technological development and automation [2]. In comparison, $24 \mathrm{~h}$ of sleeping every day corresponds to approximately 150 MET hours per week [2]. Today, most persons in developed countries spend more than half of their waking hours in seated postures which negatively affects mortality and increases the risk of, for instance, cardiovascular disease, diabetes, back pain, stroke, and obesity [3]. Hence, it is important to not only increase leisure time physical activity (PA) but also possibly increase muscle activations during sitting.

Besides leisure time PA opportunities, work places involving mainly sitting activities (e.g., office, libraries, lecture halls) represent a challenging but also promising setting for encouraging PA. In recent years, researchers have focused on active breaks, cycling desks and standing desks for promoting occupational PA [4-6]. However, despite of promising evidence such approaches have not been widely implemented. Another strategy for attaining greater PA at work, is altering the 
chair design aiming to increase "micro-activity" of the trunk while sitting [7]. Previous studies showed increased muscle activation in either thoracic or lumbar erector spinae during sitting on a stability ball [8] while performing office tasks but not for sitting on dynamic chairs [7,9]. However, meanwhile, more sophisticated dynamic chairs with moving seats have been developed. For instance, the BioSwing ${ }^{\circledR}$ chair (Haider Bioswing, Pullenreuth, Germany) enables two-dimensional swinging of the seating area. These reflective micro-motions of the trunk induced by the body are believed to beneficially affect trunk motion and muscle activity, yet to date evidence of this mechanism is lacking. Moreover, typical office activities do not only comprise individual tasks but also transitions between tasks. It has been shown that the muscle activation level during sitting is task-dependent [7]. Task transitions (i.e., from typing on the computer to calling on the phone) require more movement and can be very frequent during office works with up to 30 or 140 transitions per hour in some workers depending on the measurement method [10]. Thus, it is possible that differences in muscle activation patterns are more pronounced for tasks transitions than individual tasks. The main purpose of this study was to investigate whether dynamic sitting on a BioSwing ${ }^{\circledR}$ chair increases sway of the chair seat. Secondly, we tested the hypothesis that a greater sway of the chair induces relevant changes in trunk muscle activation during typical office tasks (reading, hand writing, calling, typing on the keyboard) and their task-transitions. Such "micro-activity" of the trunk may be particularly important when prolonged sitting cannot be avoided or when habits cannot be changed.

\section{Materials and Methods}

\subsection{Participants}

Twenty-six healthy young adults (11 males, 15 females; age $23.4 \pm 1.9$ years; body mass $65.9 \pm 10.5 \mathrm{~kg}$; height $1.73 \pm 0.08 \mathrm{~m}$; body mass index $21.9 \pm 2.6 \mathrm{~kg} / \mathrm{m}^{2}$ ) participated in this study. Written informed consent was given prior to the start of the study. Participants were blinded the chair conditions and no information on the chairs were provided. Inclusion criteria were age between 18 and 55 years of age, working most of the time in sitting position (i.e., as office workers or students), and proficiency in German language for correct and reliable introductions of the tasks. Exclusion criteria were pain, restrictions in sitting position and pregnancy. All participants were right handed and used their right hand for both writing and calling.

\subsection{Experimental Setup and Study Design}

The study design and applied procedures complied with ethical standards and were approved by the regional ethics committee (approval number: EKNZ 2016-01903). We implemented a single randomized crossover-design for sitting on two different Haider Bioswing office chairs (Figure 1). Both chairs looked identical, but in one chair, the swinging system under the seat was unlocked (dynamic chair), while in the other chair the swinging system was locked and stable (static chair). The swinging system facilitates for motion in the seat plane by employing swinging and damping elements. Both chairs were equipped with feet that did not allow motion between the chair and the floor. The participants were not aware of the differences between the chairs. 


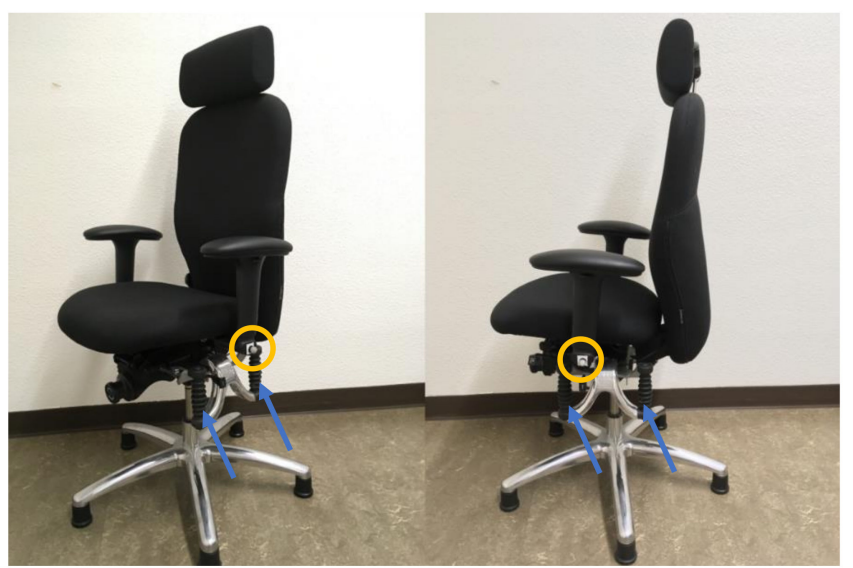

Figure 1. The dynamic swing system is located beneath the seat of the office chair (Haider Bioswing, Pullenreuth, Germany). The blue arrows indicate the location of two of four swinging elements. The yellow circles illustrate the location of the reflective markers at the base of the arm rest.

For each chair condition, participants were asked to complete four different tasks in randomized order: reading on a computer; typing on a keyboard; writing by hand; and a phone call using a mobile phone. Each task was performed for 3.5 min followed by a 30-s task-transition period where the participants were instructed for the new task. The same sequence of the four tasks was performed twice on each chair resulting in a total sitting time of $32 \mathrm{~min}$ per chair. The order of the chair conditions was randomly assigned. There was a 10-min break between testing sessions on the two chairs. Trunk muscle activity and movement of the chair were measured during the middle $30 \mathrm{~s}$ of the task and during the 30-s task-transition using surface electromyography and reflective markers (Figure 2).

Chair 1

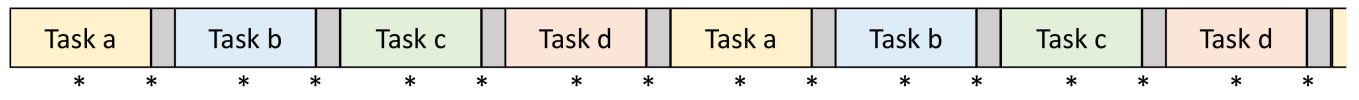

10 min break

Chair 2

\begin{tabular}{|c|c|c|c|c|c|c|c|c|c|c|c|}
\hline Task a & & Task b & & Task c & & Task d & Task a & Task b & Task c & Task d & \\
\hline$*$
\end{tabular}

Figure 2. Overview of the measurement procedures. Each start of a kinematic and EMG (electromyography) measurement is indicated with an asterisk $\left(^{*}\right)$. The grey areas between two tasks indicate the $30 \mathrm{~s}$ period of each task transition. Tasks a to d correspond to reading, calling, typing and hand writing with the order of the tasks being randomized.

All tests were performed under standardized conditions in an indoor laboratory. Chair height was adjusted for each participant to ensure full contact of the feet with the floor and a knee angle of $90^{\circ}$ and was identical between chairs.

\subsection{Tasks and Task Transitions}

For every participant, identical instructions for the tasks and task transitions were used. The task "typing on a keyboard" consisted of writing a text about the participant's current workplace (required tasks, preferences, dislikes and future possibilities). During the task "reading on a computer", participants were always asked to first open a text file and then read a text about office work. The task "writing by hand" consisted of writing a text about short-term and long-term goals and their implementation. For the task "calling", participants were instructed to enter the number on their mobile phone and to hold the phone to their ear while talking about the participants' field of work and work tasks. 


\subsection{Kinematics}

Chair motion and upper body position were measured using an 8-camera motion analysis system (Vicon, Oxford, UK). Reflective markers were placed at the base of the left and right armrest representing the chair center (Figure 2) and on the jugular notch representing the trunk. The length of the path of the midpoint between the two markers on the chair (chair center) and of the marker on the jugular notch (trunk) was calculated and used for further analysis of chair and trunk motion.

\subsection{Electromyography}

After shaving and cleaning the skin with alcohol, six wireless surface electromyography (EMG) electrodes (Myon AG, Schwarzenberg, Switzerland) were placed bilaterally on the erector spinae, obliquus internus abdominis and rectus abdominis muscles according to the guidelines of the European Surface Electromyographic Society (SENIAM) [11,12].

To remove electrocardiogram (ECG) contamination in the EMG signal of the trunk muscles, an independent component analysis based ECG filter was used [13]. All EMG signals were bandpass filtered (4th order Butterworth filter, 10-450 Hz) to remove movement artifacts and high frequency noise. EMG envelopes were calculated with a moving average filter and a window size of $41.7 \mathrm{~ms}$ (100 data points). For each participant and muscle, the mean intensity during each task and task transition was calculated. For the two chair conditions, the average of the two repetitions of each task and task transition was used for further analysis.

\subsection{Statistics}

Differences in the length of path of the chair center and mean muscle activation levels between the two chair conditions were analyzed using paired $t$-tests. Effect sizes were estimated using Cohen's d [14]. Calculations were performed in Matlab (2017a, MathWorks, Natick, MA, USA).

The change scores between both chairs were calculated as the $90 \%$ confidence intervals according to the magnitude-based inference approach [15]. A practically worthwhile change was assumed when the difference score was at least 0.2 of the between-subject standard deviation [16]. The probability for an effect being practically worthwhile was calculated according to the magnitude-based inference approach using the following scale: $25-75 \%$, possibly; $>75 \%$, likely; $>95 \%$, very likely; $>99.5 \%$, most likely [15]. The default probabilities for declaring an effect practically beneficial were $<0.5 \%$ (most unlikely) for harm and $>25 \%$ (possibly) for benefit [16]. Calculations were conducted using a Microsoft ${ }^{\circledR}$ Excel spreadsheet (Redmond, WA, USA) [17].

\section{Results}

\subsection{Chair and Trunk Motion}

On average, the length of path of the chair center for the dynamic chair was between 100 and $400 \%$ longer for the individual tasks and around 200\% greater for the task transitions compared to the static chair with a high between subject variability (Figure 3). For the separate tasks, this difference was statistically significant during calling but not during the other three tasks (Table 1). For all task transitions, this difference was statistically significant with higher effect sizes (Table 1). 


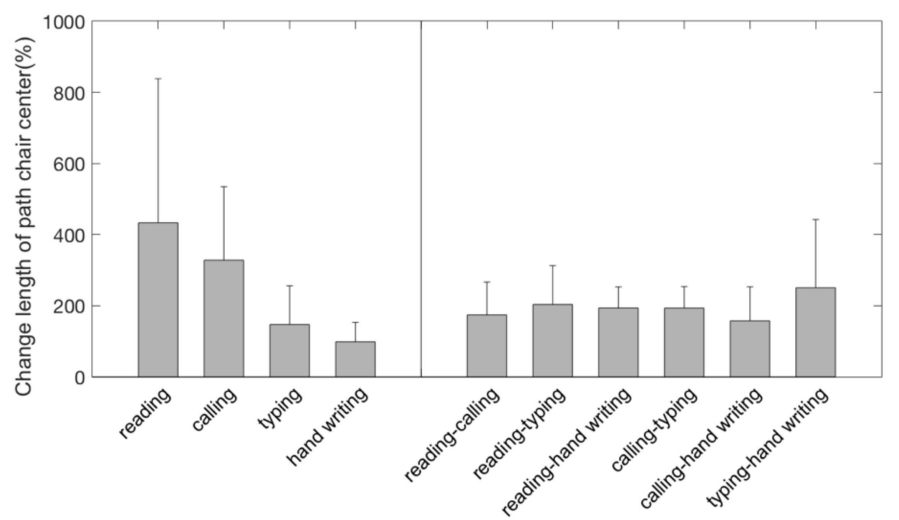

Figure 3. Relative increase in path length of the chair center for sitting on the dynamic chair compared to the static chair for the different tasks and task transitions.

Table 1. Mean (1 standard deviation) length of path of the chair center while sitting on the static or dynamic chair performing the different tasks and task transitions.

\begin{tabular}{cccccc}
\hline $\begin{array}{c}\text { Length of Path of Chair } \\
\text { Center (cm) }\end{array}$ & $\begin{array}{c}\text { Dynamic Chair } \\
\text { Mean (SD) }\end{array}$ & $\begin{array}{c}\text { Static Chair } \\
\text { Mean (SD) }\end{array}$ & $\boldsymbol{p}$-Value & Effect Size & MBI \\
\hline Tasks & & & & & \\
Reading & $1.2(1.7)$ & $0.6(0.8)$ & 0.111 & 0.444 & $\begin{array}{c}\text { likely } \\
\text { Calling }\end{array}$ \\
Typing & $6.0(5.6)$ & $3.8(4.4)$ & 0.003 & 0.446 & most likely \\
Hand writing & $1.2(1.4)$ & $0.7(0.7)$ & 0.111 & 0.401 & likely \\
Task transitions & $0.9(1.0)$ & $0.6(0.6)$ & 0.096 & 0.349 & likely \\
Reading-calling & & & & & \\
Reading-typing & $6.9(5.4)$ & $3.6(2.6)$ & 0.025 & 0.767 & most likely \\
Reading-hand writing & $9.5(5.1)$ & $1.7(1.4)$ & 0.016 & 0.745 & most likely \\
Calling-typing & $8.6(5.8)$ & $4.1(3.1)$ & $<0.001$ & 1.150 & most likely \\
Calling-hand writing & $4.5(4.2)$ & $4.3(3.6)$ & 0.004 & 0.875 & most likely \\
Typing-hand writing & $7.2(5.6)$ & $2.0(1.4)$ & 0.012 & 0.800 & likely \\
\hline
\end{tabular}

SD—standard deviation; MBI—-magnitude based inferences: the probability of a beneficial effect towards the dynamic chair.

The length of path of the trunk was on average shorter on the dynamic compared to the static chair during hand writing $(p=0.041$, effect size $=0.349)$. For the other tasks and all task transitions, there were no significant differences in the length of path between the chairs (Figure 4).

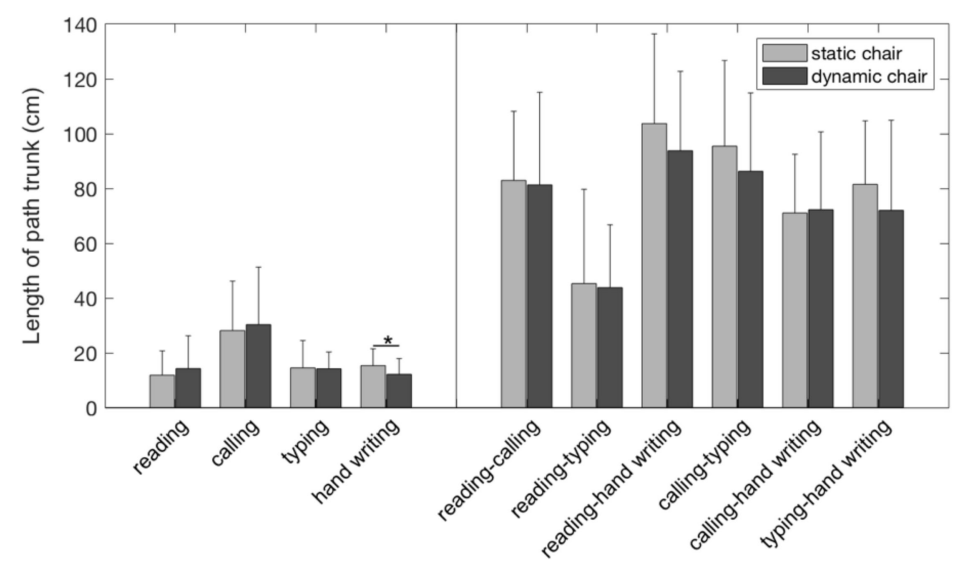

Figure 4. Length of path of the trunk motion during the different tasks and task transitions while sitting on the dynamic (dark grey) and static chair (light grey). The asterisk indicates $\left(^{*}\right)$ significant differences between the chair conditions $(p<0.05)$. 


\subsection{Muscle Activation}

\subsubsection{Tasks}

Mean muscle intensities while performing the different tasks did not differ between the dynamic and static chairs. Yet for hand writing, the left obliquus internus muscle showed a possible effect with lower mean intensity on the dynamic chair than on the static chair with only a small effect $(p=0.066$; effect size $=-0.188$; Table 2).

Table 2. Mean (1 standard deviation) mean muscle intensity while sitting on the static or dynamic chair performing the different tasks.

\begin{tabular}{|c|c|c|c|c|c|}
\hline $\begin{array}{c}\text { Mean Intensity of } \\
\text { Muscle (mV) }\end{array}$ & $\begin{array}{c}\text { Dynamic Chair } \\
\text { Mean (SD) }\end{array}$ & $\begin{array}{l}\text { Static Chair } \\
\text { Mean (SD) }\end{array}$ & $p$-Value & Effect Size & MBI \\
\hline \multicolumn{6}{|l|}{ Reading } \\
\hline Rectus abdominis right & $14.4(4.4)$ & $14.2(5.0)$ & 0.824 & 0.044 & trivial \\
\hline Obliquus internus right & $14.1(4.6)$ & $13.9(4.8)$ & 0.598 & 0.052 & trivial \\
\hline Erector spinae right & $14.2(5.0)$ & $14.1(5.6)$ & 0.951 & 0.005 & trivial \\
\hline Rectus abdominis left & $13.8(4.1)$ & $13.7(4.5)$ & 0.919 & 0.010 & trivial \\
\hline Obliquus internus left & $14.1(4.4)$ & $14.4(4.6)$ & 0.613 & -0.058 & trivial \\
\hline Erector spinae left & $13.9(5.0)$ & $14.2(5.7)$ & 0.501 & -0.054 & trivial \\
\hline \multicolumn{6}{|l|}{ Calling } \\
\hline Rectus abdominis right & $14.6(5.0)$ & $14.1(4.8)$ & 0.677 & 0.101 & trivial \\
\hline Obliquus internus right & $14.6(4.6)$ & $14.7(5.0)$ & 0.858 & -0.014 & trivial \\
\hline Erector spinae right & $13.8(4.7)$ & $14.3(5.3)$ & 0.292 & -0.105 & trivial \\
\hline Rectus abdominis left & $13.5(4.7)$ & $14.0(5.1)$ & 0.243 & -0.101 & trivial \\
\hline Obliquus internus left & $15.5(4.6)$ & $15.2(4.6)$ & 0.483 & 0.073 & trivial \\
\hline Erector spinae left & $14.3(6.2)$ & $15.1(7.2)$ & 0.380 & -0.124 & trivial \\
\hline \multicolumn{6}{|l|}{ Typing } \\
\hline Rectus abdominis right & $14.1(4.0)$ & $14.0(4.9)$ & 0.863 & 0.021 & trivial \\
\hline Obliquus internus right & $14.3(4.5)$ & $14.5(4.8)$ & 0.648 & -0.036 & trivial \\
\hline Erector spinae right & $15.3(6.3)$ & $15.7(6.2)$ & 0.699 & -0.058 & trivial \\
\hline Rectus abdominis left & $14.0(4.6)$ & $13.8(4.8)$ & 0.716 & 0.042 & trivial \\
\hline Obliquus internus left & $14.4(4.4)$ & $14.6(4.4)$ & 0.655 & -0.041 & trivial \\
\hline Erector spinae left & $15.3(7.0)$ & $15.0(6.7)$ & 0.689 & 0.042 & trivial \\
\hline \multicolumn{6}{|l|}{ Hand writing } \\
\hline Rectus abdominis right & $13.9(4.1)$ & $14.2(4.9)$ & 0.421 & -0.078 & trivial \\
\hline Obliquus internus right & $14.3(4.3)$ & $14.8(4.7)$ & 0.131 & -0.111 & trivial \\
\hline Erector spinae right & $14.3(5.0)$ & $14.8(5.5)$ & 0.193 & -0.105 & trivial \\
\hline Rectus abdominis left & $15.3(8.7)$ & $14.6(4.8)$ & 0.567 & 0.097 & trivial \\
\hline Obliquus internus left & $14.7(4.2)$ & $15.4(4.1)$ & 0.066 & -0.188 & possibly \\
\hline Erector spinae left & $15.7(7.4)$ & $16.0(7.5)$ & 0.603 & -0.042 & trivial \\
\hline
\end{tabular}

\subsubsection{Task transitions}

For the transition from reading to calling, the mean intensity of the left erector spinae was likely higher for the dynamic chair than the static chair $(p=0.067$; effect size $=0.332)$. For the transition from calling to hand writing, the mean intensity of the right erector spinae was likely lower for the dynamic than the static chair $(p=0.036$; effect size $=-0.192)$. Moreover, there was a possibly lower mean intensity of the right erector spinae during the transition from reading to hand writing and typing to hand writing, of the left obliquus internus during the transition from calling to hand writing, of the left rectus abdominis during the transition from reading to typing, and of the right rectus abdominis during the transition from calling to hand writing for the dynamic chair compared to the static chair. All observed effect sizes were small (Table 3). 
Table 3. Mean (SD) mean muscle intensity while sitting on the two chairs during the different task transitions.

\begin{tabular}{|c|c|c|c|c|c|}
\hline $\begin{array}{l}\text { Mean Intensity of } \\
\text { Muscle (mV) }\end{array}$ & $\begin{array}{c}\text { Dynamic Chair } \\
\text { Mean (SD) }\end{array}$ & $\begin{array}{l}\text { Static Chair } \\
\text { Mean (SD) }\end{array}$ & $p$-Value & Effect Size & MBI \\
\hline \multicolumn{6}{|l|}{ Reading to calling } \\
\hline Rectus abdominis right & $13.7(4.5)$ & $13.0(5.3)$ & 0.530 & 0.136 & trivial \\
\hline Obliquus internus right & $13.7(4.9)$ & $13.6(5.4)$ & 0.844 & 0.020 & trivial \\
\hline Erector spinae right & $16.3(5.5)$ & $15.5(5.9)$ & 0.336 & 0.132 & trivial \\
\hline Rectus abdominis left & $13.5(5.0)$ & $13.8(6.0)$ & 0.609 & -0.055 & trivial \\
\hline Obliquus internus left & $14.7(4.6)$ & $13.8(5.3)$ & 0.224 & 0.179 & trivial \\
\hline Erector spinae left & $17.0(6.5)$ & $15.0(5.8)$ & 0.067 & 0.332 & likely \\
\hline \multicolumn{6}{|l|}{ Reading to typing } \\
\hline Rectus abdominis right & $13.5(4.7)$ & $14.0(5.0)$ & 0.374 & -0.095 & trivial \\
\hline Obliquus internus right & $14.1(4.8)$ & $14.5(5.1)$ & 0.383 & -0.078 & trivial \\
\hline Erector spinae right & $14.6(5.4)$ & $15.3(5.9)$ & 0.251 & -0.117 & unclear \\
\hline Rectus abdominis left & $13.0(4.5)$ & $14.0(4.9)$ & 0.130 & -0.199 & possibly \\
\hline Obliquus internus left & $14.5(4.1)$ & $14.7(5.0)$ & 0.658 & -0.054 & trivial \\
\hline Erector spinae left & $14.4(6.2)$ & $15.1(5.5)$ & 0.317 & -0.111 & trivial \\
\hline \multicolumn{6}{|l|}{ Reading to hand writing } \\
\hline Rectus abdominis right & $15.4(1.9)$ & $15.9(4.5)$ & 0.591 & -0.143 & trivial \\
\hline Obliquus internus right & $15.7(3.0)$ & $16.1(3.7)$ & 0.417 & -0.120 & trivial \\
\hline Erector spinae right & $17.9(5.0)$ & $18.9(4.4)$ & 0.139 & -0.220 & possibly \\
\hline Rectus abdominis left & $16.0(6.1)$ & $15.5(4.1)$ & 0.842 & 0.090 & trivial \\
\hline Obliquus internus left & $16.3(2.7)$ & $16.6(3.1)$ & 0.468 & -0.098 & trivial \\
\hline Erector spinae left & $17.7(5.9)$ & $18.5(4.9)$ & 0.361 & -0.162 & trivial \\
\hline \multicolumn{6}{|l|}{ Calling to typing } \\
\hline Rectus abdominis right & $16.3(3.6)$ & $15.4(4.0)$ & 0.554 & 0.247 & trivial \\
\hline Obliquus internus right & $15.5(3.1)$ & $15.9(3.8)$ & 0.461 & -0.106 & trivial \\
\hline Erector spinae right & $18.4(6.3)$ & $18.0(4.6)$ & 0.571 & 0.081 & trivial \\
\hline Rectus abdominis left & $15.4(5.6)$ & $14.8(4.1)$ & 0.677 & 0.132 & trivial \\
\hline Obliquus internus left & $15.9(2.5)$ & $16.1(3.4)$ & 0.810 & -0.041 & trivial \\
\hline Erector spinae left & $19.4(7.8)$ & $18.9(5.8)$ & 0.620 & 0.073 & trivial \\
\hline \multicolumn{6}{|l|}{ Calling to hand writing } \\
\hline Rectus abdominis right & $13.6(4.3)$ & $15.2(6.6)$ & 0.121 & -0.281 & possibly \\
\hline Obliquus internus right & $14.5(4.4)$ & $14.8(4.8)$ & 0.217 & -0.078 & unclear \\
\hline Erector spinae right & $14.4(5.6)$ & $15.5(5.8)$ & 0.036 & -0.192 & likely \\
\hline Rectus abdominis left & $13.6(4.2)$ & $14.1(4.4)$ & 0.227 & -0.120 & unclear \\
\hline Obliquus internus left & $15.0(4.0)$ & $15.6(4.6)$ & 0.150 & -0.124 & possibly \\
\hline Erector spinae left & $16.1(5.7)$ & $15.8(6.3)$ & 0.642 & 0.053 & trivial \\
\hline \multicolumn{6}{|l|}{ Typing to hand writing } \\
\hline Rectus abdominis right & $13.4(4.6)$ & $13.4(5.5)$ & 0.978 & -0.004 & trivial \\
\hline Obliquus internus right & $13.7(5.0)$ & $14.3(5.5)$ & 0.247 & -0.118 & unclear \\
\hline Erector spinae right & $16.3(5.8)$ & $17.6(6.3)$ & 0.116 & -0.207 & possibly \\
\hline Rectus abdominis left & $13.1(5.2)$ & $14.2(5.8)$ & 0.213 & -0.192 & unclear \\
\hline Obliquus internus left & $14.3(4.7)$ & $14.7(4.8)$ & 0.431 & -0.088 & trivial \\
\hline Erector spinae left & $16.2(6.1)$ & $16.6(6.1)$ & 0.550 & -0.073 & trivial \\
\hline
\end{tabular}

\section{Discussion}

The aim of this study was to investigate whether a BioSwing ${ }^{\circledR}$ office chair induces changes in chair motion and trunk muscle activity compared to a static non-swinging chair in a single blinded randomized controlled crossover trial. Our results indicate that (a) the path length of the chair center was significantly longer for the dynamic chair during calling and all task transitions and (b) muscle 
activation during all tasks did not differ between the chairs but showed small to notable changes during the task transitions reading to calling, calling to writing and typing to writing. Specifically, for the transition between reading and calling bilateral erector spinae muscle activation was higher for the dynamic chair than for the static chair but effect sizes were small and MBI revealed only a trivial effect. For the transitions between calling and writing and between typing and writing the right erector spinae muscle activation was lower for the dynamic chair than for the static chair with very small effect sizes and trivial MBI.

\subsection{Chair Center}

The longer path length of the chair center for the dynamic than for the static chair confirms that the springs facilitate motion of the seat. This is comparable to previous results with a different dynamic office chair that showed greater chair movements (measured on the top of the backrest) when performing office tasks such as typing on a keyboard and placing files in folders while sitting on a dynamic compared to a conventional chair [18]. During all measurements, participants had their feet on the floor, which typically has a stabilizing effect on the body especially for more static tasks such as writing by hand, typing and reading on the laptop screen where the arms are also placed on the desk. Thus, limited movement of the hips and buttocks might result in negligible changes in chair movement and trunk muscle activation. However, motion analyses of the hip and buttocks were not explicitly conducted. During the condition calling, participants moved more freely on the chair, often sitting back in the chair and not placing their arms on the desk thereby increasing the degrees of freedom of possible motion which has been shown to increase postural sway [19]. Moreover, this might allow more micro-motions of the seat of the dynamic chair than the static chair resulting in a longer path length of the chair center. Similarly, when performing task transitions, participants often had to remove their arms from the desk and move in the chair enabling the swinging of the seating area as seen in the longer path length of the chair center for the dynamic chair than for the static chair.

\subsection{Muscle Activation}

Previous studies on trunk muscle activation during sitting on different chairs reported different changes in muscle activation levels depending on the design of the tested chairs and measured muscles. No differences in lumbar and thoracic erector spinae activation were reported for reading a book, word-processing and computer-aided design between sitting on a chair with fixed seat and backrest, a chair which allowed independent sagittal plane rotation of the seat and back rest and a chair which allowed a rotation in a fixed ratio between the seat and back rest [7]. Similarly, Ellegast et al. did not observe an effect of sitting on five different office chairs on erector spinae muscle activation during different tasks such as typing, calling, and reading and correcting text data [9]. However, the office chairs investigated in these studies differed from the office chairs tested in our study. Nonetheless, it is possible that the variability in performing different office tasks between trials and persons is higher than the difference in the construction of the office chairs. On the contrary, sitting on a stability ball resulted on average in a higher activation of the left thoracic erector spinae muscle during reading, computer-aided design and typing than sitting on a standard office chair without armrests [8]. However, participants in that study also reported an increased level of discomfort, which might be related to altered posture or muscle activations. An ergonomic backless chair resulted in a significantly lower activation of the iliocostalis pars thoracis muscle for a typing task compared to a standard backless office chair [12]. In a systematic review, it was concluded that the changes in muscle activation levels during dynamic sitting may be attributable to the absence of a backrest in some dynamic chairs [20]. However, because the BioSwing ${ }^{\circledR}$ chair used in our study has a backrest, the observed changes in muscle activation are likely attributable to the dynamic swinging system.

Generally, muscle activation levels during seated office tasks were small (below $10 \%$ of maximal voluntary contraction) in all studies [7,9]. Although we did not normalize muscle activation levels to maximal voluntary contractions but assessed change scores, visual inspections of the EMG signals 
showed that the signals remained close to baseline. None of the previous studies [20] reported effects of the different chairs on the activation of ventral trunk muscles (rectus abdominis, external oblique, internal oblique), which was confirmed for individual tasks for the dynamic BioSwing ${ }^{\circledR}$ chair in our study. In contrast to these previous studies [20], our study showed differences in trunk muscle activations during task transitions. Task transitions comprise the combination of two tasks and include more movement and variability because the participants also rearranged the work space (i.e., moving laptop closer or away, picking up the phone). This may explain the small observed changes in the trunk muscles and mainly the erector spinae muscle. We are not aware of previous studies investigating task transitions, but effects of different office tasks on muscle activation levels during sitting have been reported $[8,9]$.

Contrary to our initial hypothesis, we observed mainly possible or likely lower trunk muscle activity during task transitions while sitting on the dynamic compared to the static office chair. This result is in contrast to a previous study that showed an increase in the thoracic erector spinae muscle activation for sitting on an unstable ball compared to a conventional chair [8] but agrees with results from another study reporting lower activation of the iliocostalis pars thoracis muscle for a dynamic chair than a static chair without backrests [12]. It is possible that the swinging system of the dynamic chair facilitates lumbar trunk movements, thus reducing muscle activation levels and delaying a possible onset of fatigue.

\subsection{Strengths and Limitations}

The tested dynamic and static chairs in this study were identical except of the swinging mechanism being enabled for the dynamic and disabled for the static chair. Moreover, participants were blinded to the differences between chairs before the experiment to reduce potential conscious adaptions in sitting posture and movements during the measurements. To reflect common working situations, we not only investigated muscle activations during the performance of single tasks but also during task transitions. The randomization of the order of the tasks resulted in 12 possible task transitions with not every participant performing the same task transitions. To increase the number of observations per task transition, both directions of the task transition (i.e., calling to typing and typing to calling) were pooled for the analysis. This might have increased the variability in muscle activation patterns but should have little effects on mean muscle intensity over the entire 30-s measurement. Nevertheless, it is possible that the data pooling reduced the observed differences and resulted in smaller differences between chair conditions for task transitions. In this study, we tested the experimental chairs in 26 subjects. Similar results would have to be confirmed in a larger population. Moreover, the study population consisted of healthy young adults, and hence it is not clear if older adults or persons with back problems would experience similar differences. Nonetheless, the data presented in this study represents reference data for future studies on the effect of office chair design on trunk muscle activity chair motion.

\section{Conclusions}

The increase in the path length of the chair center of the dynamic chair indicates changes in the chair motion of the dynamic chair compared to those of the static chair. However, the observed altered chair motion was associated with only small differences in trunk muscle activation levels for sitting on the dynamic chair compared to the static chair. In addition to previous studies, not only office tasks but also tasks transitions were investigated and it was seen that there are more changes in trunk muscle activation levels during these task transitions than for the individual tasks. Hence, investigating task transitions might be more sensitive in assessing effects of dynamic chairs on muscle activation.

Greater changes in muscle activation levels may be achieved when removing the stabilizing effect of placing the feet on the floor by adding a foot rest facilitating swinging of the whole body. The effect of the dynamic chair on chair motion and muscle activity levels may depend on the stiffness of the swinging elements, and it may be necessary to adjust the stiffness of the swinging elements to the 
user's body weight. Nonetheless, from an epidemiological perspective even small changes in muscle activity during office tasks and task transition may be meaningful in the long run considering that many professionals perform mainly seated tasks during their work life over decades.

Author Contributions: C.N., J.-N.K., A.M. and L.D. designed the study; J.-N.K. recruited the participants and collected the data; C.N. prepared the data for statistical analysis; C.N., A.M. and L.D. performed the statistical analysis. C.N., J.-N.K. and L.D. wrote the manuscript; C.N., J.-N.K., A.M. and L.D. reviewed and revised the manuscript.

Funding: This study was partly funded by Haider Bioswing.

Acknowledgments: We are grateful for the participation and compliance of all study participants. Moreover, we would like to thankfully acknowledge Haider Bioswing, who provided the chairs and supported a sound and independent investigation without influencing the study protocol and publishing process with the intention to further improve their product based on scientific evidence.

Conflicts of Interest: The funders had no role in the design of the study; in the collection, analyses, or interpretation of data; in the writing of the manuscript, or in the decision to publish the results.

\section{References}

1. Blair, S.N. Physical inactivity: The biggest public health problem of the 21st century. Br. J. Sports Med. 2009, 43, 1-2. [PubMed]

2. Ng, S.W.; Popkin, B.M. Time use and physical activity: A shift away from movement across the globe. Obes. Rev. 2012, 13, 659-680. [CrossRef] [PubMed]

3. Hamilton, M.T.; Hamilton, D.G.; Zderic, T.W. Role of Low Energy Expenditure and Sitting in Obesity, Metabolic Syndrome, Type 2 Diabetes, and Cardiovascular Disease. Diabetes 2007, 56, 2655-2667. [CrossRef] [PubMed]

4. Faude, O.; Donath, L.; Bopp, M.; Hofmann, S.; Erlacher, D.; Zahner, L. Neuromuscular training in construction workers: A longitudinal controlled pilot study. Int. Arch. Occup. Environ. Health 2015, 88, 697-705. [CrossRef] [PubMed]

5. Joubert, L.; Kilgas, M.; Riley, A.; Gautam, Y.; Donath, L.; Drum, S. In-Class Cycling to Augment College Student Academic Performance and Reduce Physical Inactivity: Results from an RCT. Int. J. Environ. Res. Public Health 2017, 14, 1343. [CrossRef] [PubMed]

6. Wick, K.; Faude, O.; Manes, S.; Zahner, L.; Donath, L. I Can Stand Learning: A Controlled Pilot Intervention Study on the Effects of Increased Standing Time on Cognitive Function in Primary School Children. Int. J. Environ. Res. Public Health 2018, 15, 356. [CrossRef] [PubMed]

7. Van Dieen, J.; De Looze, M.; Hermans, V. Effects of dynamic office chairs on trunk kinematics, trunk extensor EMG and spinal shrinkage. Ergonomics 2001, 44, 739-750. [CrossRef] [PubMed]

8. Gregory, D.E.; Dunk, N.M.; Callaghan, J.P. Stability ball versus office chair: Comparison of muscle activation and lumbar spine posture during prolonged sitting. Hum. Factors 2006, 48, 142-153. [CrossRef] [PubMed]

9. Ellegast, R.P.; Kraft, K.; Groenesteijn, L.; Krause, F.; Berger, H.; Vink, P. Comparison of four specific dynamic office chairs with a conventional office chair: Impact upon muscle activation, physical activity and posture. Appl. Ergon. 2012, 43, 296-307. [CrossRef] [PubMed]

10. Van Eerd, D.; Hogg-Johnson, S.; Mazumder, A.; Cole, D.; Wells, R.; Moore, A. Task exposures in an office environment: A comparison of methods. Ergonomics 2009, 52, 1248-1258. [CrossRef] [PubMed]

11. Hermens, H.J.; Freriks, B.; Disselhorst-Klug, C.; Rau, G. Development of recommendations for SEMG sensors and sensor placement procedures. J. Electromyogr. Kinesiol. 2000, 10, 361-374. [CrossRef]

12. O'Sullivan, K.; McCarthy, R.; White, A.; O'Sullivan, L.; Dankaerts, W. Lumbar posture and trunk muscle activation during a typing task when sitting on a novel dynamic ergonomic chair. Ergonomics 2012, 55, 1586-1595. [CrossRef] [PubMed]

13. Willigenburg, N.W.; Daffertshofer, A.; Kingma, I.; van Dieën, J.H. Removing ECG contamination from EMG recordings: A comparison of ICA-based and other filtering procedures. J. Electromyogr. Kinesiol. 2012, 22, 485-493. [CrossRef] [PubMed]

14. Cohen, J. A power primer. Psychol. Bull. 1992, 112, 155. [CrossRef] [PubMed]

15. Batterham, A.M.; Hopkins, W.G. Making meaningful inferences about magnitudes. Int. J. Sports Physiol. Perform. 2006, 1, 50-57. [CrossRef] [PubMed] 
16. Hopkins, W.; Marshall, S.; Batterham, A.; Hanin, J. Progressive statistics for studies in sports medicine and exercise science. Med. Sci. Sport. Exerc. 2009, 41, 3. [CrossRef] [PubMed]

17. Hopkins, W.G. A Spreadsheet for Deriving a Confidence Interval, Mechanistic Inference and Clinical Inference from a p Value. Sportscience 2007, 11, 16-20.

18. Grooten, W.J.A.; Äng, B.O.; Hagströmer, M.; Conradsson, D.; Nero, H.; Franzén, E. Does a dynamic chair increase office workers' movements?-Results from a combined laboratory and field study. Appl. Ergon. 2017, 60, 1-11. [CrossRef] [PubMed]

19. Hendriks, H.M.; Spoor, C.W.; de Jong, A.M.; Goossens, R.H.M. Stability of sitting postures: The influence of degrees of freedom. Ergonomics 2006, 49, 1611-1626. [CrossRef] [PubMed]

20. O'Sullivan, K.; O'Sullivan, P.; O'Keeffe, M.; O'Sullivan, L.; Dankaerts, W. The effect of dynamic sitting on trunk muscle activation: A systematic review. Appl. Ergon. 2013, 44, 628-635. [CrossRef] [PubMed]

2018 by the authors. Licensee MDPI, Basel, Switzerland. This article is an open access article distributed under the terms and conditions of the Creative Commons Attribution (CC BY) license (http://creativecommons.org/licenses/by/4.0/). 